\title{
Computer graphic representation of mandibular movements in three dimensions: the method
}

JOHN G. KNAPP The University of Michigan, School of Dentistry, Ann Arbor, MI, U.S.A.

\section{Summary}

A mathematical simulation of the Denar D4A articulator and a simulated complement of graphically represented teeth were programmed in a Prime 4000 minicomputer. The computer program could be manipulated to quantitatively evaluate the theoretical effects of changes in articulator and control factors on the anatomy of selected teeth or tooth groups. The effects of these changes could be assessed graphically and numerically and data could be manipulated statistically.

\section{Introduction}

The study of the jaw movements is often of reduced significance unless the effects of movement can be related to tooth anatomy. Investigators, attempting to study mandibular movements and associated tooth anatomy, used articulators which mechanically simulated jaw movement (Mjor, 1965; Aull, 1965). Their tracings were made using styli and recording tables placed at the approximate position of the tooth. When movements were made, recordings in three planes allowed visualization and measurement of the aspects of occlusal anatomy that correlated to articulator end control factors. Unfortunately, these analytical methods were cumbersome and limited in accuracy. Repetitive samples were required to provide average recordings for each movement in an attempt to reduce both the effects of human error in observation and measurement, and the effects of the mechanical inconsistencies. Tedious labour and exhaustive statistical analysis was required. The purpose of this project was to provide a reproducible description of a simulated tooth and to relate this tooth to the movements of a simulated articulator.

\section{Method}

\section{Computer-graphics systems}

A computer-graphics system has previously been described which computed mandibular movements effected by various articulator settings and graphically plotted the resulting interrelationships between the maxillary and mandibular teeth (Roedema et al., 1978,a,b). The Denar D5A* articulator was the mechanical model upon which

* Denar Corporation, Anaheim, Califor nia.

Correspondence: Dr John Knapp, 19928 Farmington, Livonia, Michigan 48152, U.S.A.

$$
\text { 0305-182X/83/0700-0295\$02.00 } \quad \text { (C) } 1983 \text { Blackwell Scientific Publications }
$$




\begin{tabular}{l|l|l|l|l}
$\begin{array}{l}\text { Coordinates and } \\
\text { Direction of (t) }\end{array}$ & Definition & Abbreviation & Initial Settings & Limits \\
\hline Value
\end{tabular}

Fig. 1. The articulator end control factors: their abbreviations, the directions of positive value adjustment, initial settings, and the limits of the range of adjustment are specified to enhance clarity.

the computerized mathematical model was based. Articulator end control factors were carefully defined with respect to convention of movement and limits of adjustment (Fig 1). The computer program was prepared using the mathematical model and was entered onto a Prime model 400 computer.* Simulated opposing complements of teeth, detailed in three views, were also entered into the computer program. The simulation could display the effects of mandibular movement patterns of both the maxillary and mandibular teeth in the frontal, sagittoid and occlusal planes. The parameters affecting mandibular movement patterns could be varied selectively.

Additional programming permitted greater flexibility in the analysis of the simula-

* Prime Computer, Inc., Framingham, Massachusetts. 
tion of the occlusal surface of the mandibular left first molar and the opposing mesiolingual cusp of the maxillary first molar.

\section{The mandibular left first molar and its position}

The origin of the reference coordinate system was the centre of the left condyle. Fourteen teeth in each of the maxillary and mandibular arches of teeth were simulated and digitized, each arch being bilaterally symmetrical about the mid-sagittal plane. The plane of occlusion was placed $33.0 \mathrm{~mm}$ below the centre of the condyles and parallel to this horizontal $X-Y$ plane. The cusps of the mandibular teeth touched the occlusal plane with the fossae $1.5 \mathrm{~mm}$ below this plane. The lingual cusps of the maxillary teeth occluded in the appropriate mandibular fossae, and thus, were $34.5 \mathrm{~mm}$ below the centre of the condyles. The incisor teeth were placed $85 \mathrm{~mm}$ anterior to the condylar axis. The initial setting of the central fossae of the mandibular left first molar was $53.8 \mathrm{~mm}$ along the $Y$ and $28.01 \mathrm{~mm}$ along the $X$ axis (Fig. 2). At the option

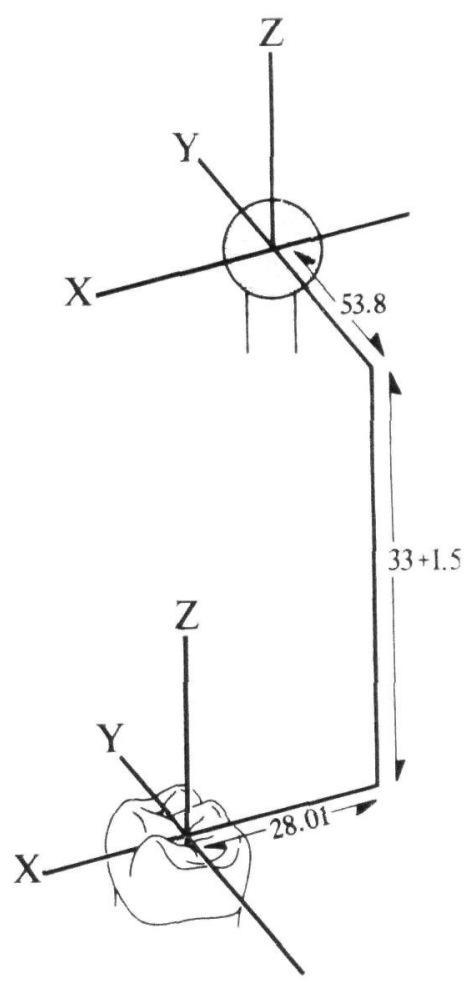

Fig. 2. The relationship of the central fossae of tooth No.19 to the centre of the left condyle of the articulator is designated relative to identical coordinate axis.

of the researcher, these initial values could readily be changed and the occlusal plane rotated or translated as a unit to other coordinate values within the confines of the simulated articulator.

\section{The angles measured}

The angles of interest were measured relative to several projection planes; horizontal frontal and sagittal were used for reference. Pathways of movement were viewed perpendicular to these coordinate planes as if the observer were outside the articulator simulation looking from the anatomic top, front or side. Angles formed by the projected view of the arc of movement and the coordinate plane were called coordinate plane angles. In Fig. 3a, the observer looks at the horizontal projection of the sample 

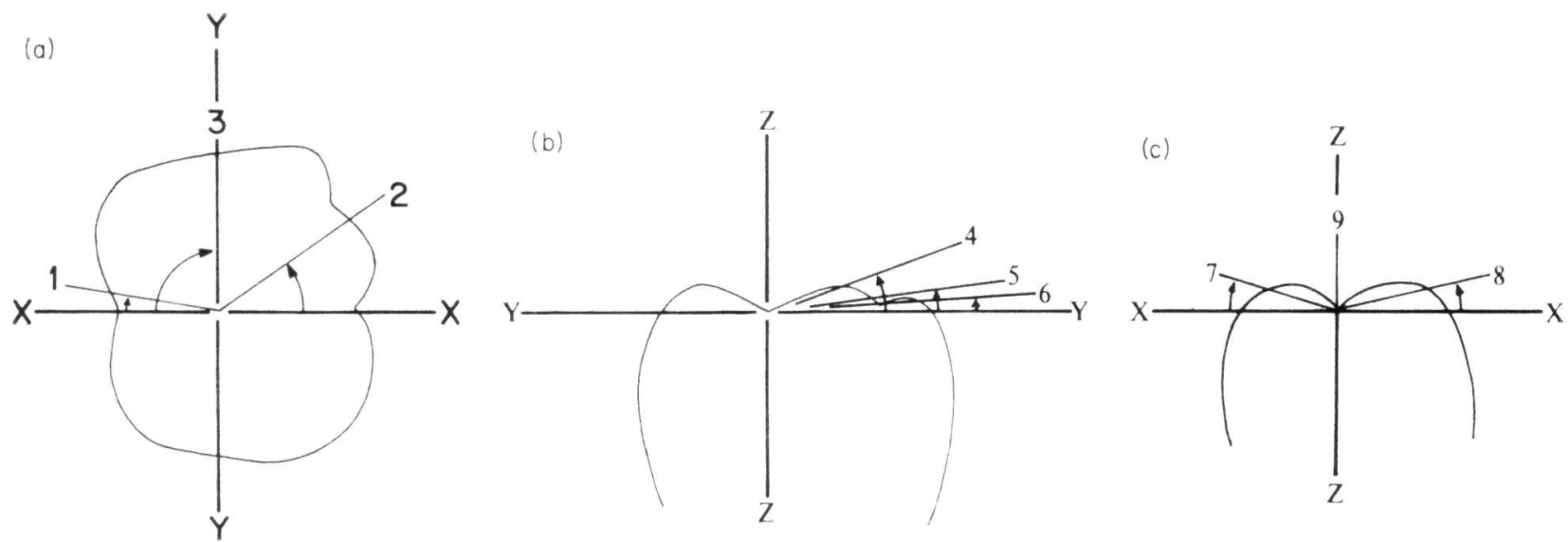

Fig. 3. Views generated by the computer in the occlusal, sagittoid, and frontal planes are related tc the coordinates. The angle of mandibular movement pathways are measured from the coordinate to yield data with positive values. These schematics show a representation of relative direction of positive values: (a) the horizontal surface in the $X-Y$ plane; (b) the sagittal cross section in the $X-Y$ plane; (c) the frontal cross section in the $X-Y$ plane.

tooth with the central fossa in the plane of occlusion congruent with the coordinate plane. This projection of the $X-Y$ plane shows a sample of projected arcs of movement, their relationships to the coordinate planes and the convention of measurement of the positive value used in tabulating data. Similarly, in Fig. 3b and c, the observer would be looking at the respective sagittoid and frontal planes, the tooth projection location, and samples of projected arcs of movement. They also show the relationships to coordinate planes and the conventions of positive value measurement.

This study also measured the groove elevation angle, or the smallest angle formed by the arc of movement and the $X-Y$ plane. This view was not projected to a cordinated plane but to the plane formed by the arc of movement and the horizontal plane. Table 1 lists the selection of angles measured and the appropriate identifying sign.

\section{Volume}

The various articulator settings and tooth positional dimensions naturally may influence the configurations of an occlusal surface. The results of these parameter

Table 1. The menu of the selection of angles and volume was presented by the computer terminal using the designated identifying sign

\begin{tabular}{ll}
\hline $\mathrm{L}$ & Left working groove elevation angle \\
$\mathrm{R}$ & Right working groove elevation angle \\
$\mathrm{P}$ & Protrusive groove elevation angle \\
1 & Left working, horizontal coordinate plane angle \\
2 & Right working, horizontal coordinate plane angle \\
3 & Protrusive, horizontal coordinate plane angle \\
4 & Left working, sagittal coordinate plane angle \\
5 & Right working, sagittal coordinate plane angle \\
6 & Protrusive, sagittal coordinate plane angle \\
7 & Left working, frontal coordinate plane angle \\
8 & Right working, frontal coordinate plane angle \\
9 & Protrusive, frontal coordinate plane angle \\
$<$ & Left working, volume \\
$>$ & Right working, volume \\
\hline
\end{tabular}




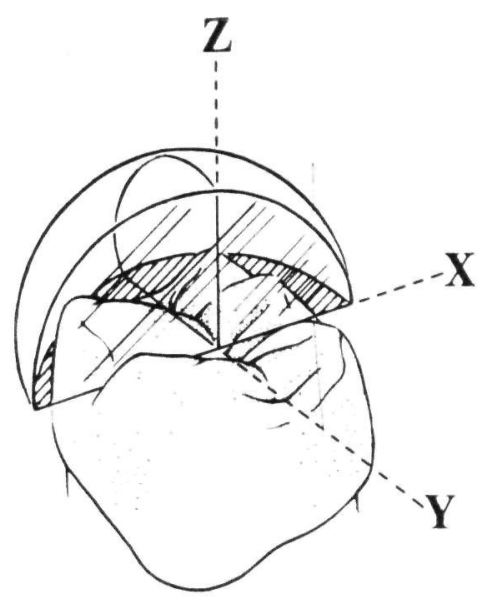

Fig. 4. The hemisphere used to represent the potential tooth volume and its relationship to the central fossae of tooth No.19 is never graphically illustrated by the computer terminal. This figure is a rendition of the concept, superimposing the coordinate relationship between the tooth and the hemisphere.

changes were qualified as volumetric changes of the tooth. The figure used to represent the tooth volume was $2.5 \mathrm{~mm}$ radius hemisphere whose flat surface was congruent with the horizontal plane of the mandibular fossae (Fig. 4). This hemisphere was intersected by four lines originating at the spherical centre, the central fossa of the lower left first molar. These lines represented arcs of movement and reflected the relative movement of the mandibular tooth in relationship to the lingual cusp of the opposing maxillary tooth. One pair of lines was used as a reference plane with respect to the other pair of lines. The reference set generated by use of standard settings consisted of a protrusive path and either the left or right working path, depending on the excursive movement selected by the operator. The variable pair of lines consisted of a corresponding protrusive path and a left or right working path, both produced by the experimental settings entered into the simulation. These four lines in space, each intersecting with the periphery of the hemisphere, formed a solid which resembled a pyramid (Fig. 5). The apex was the origin, or central fossa, while the base was the portion of the hemisphere defined by the intersection of the pair of reference lines and the pair of experimental lines of movement. The lines forming the solid of interest

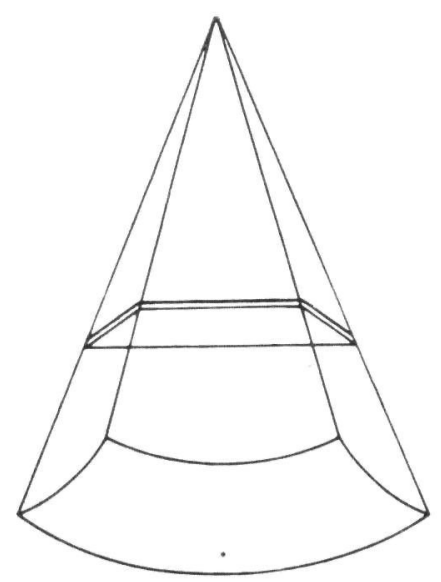

Fig. 5. This figure illustrates the theoretical pyramidal sphere section formed by the two pairs of lines originating at the central fossa and intersecting the spherical surface. Also shown is the prismatoids used for subdividing and measuring the volume of the sphere section. 
were not necessarily straight, but in certain instances curved slightly from the apex to base.

The method of analysing this solid was to approximate it by sectioning it into numerous parallel prismatoids with quadrilateral bases and trapezoidal lateral faces. These sections were perpendicular to the centroid of the solid. The volume for each of these prismatoids was calculated by the standard equation:

$$
V=1 / 6 h(B 1+4 M+B 2)
$$

where $B 1$ was the area of the lower base, $M$ the area of the midsection, $B 2$ the area of the upper base, and $h$ the height or thickness of the prismatoid.

In calculating the volume of the solid, two approximations were found to be necessary. The first was that the paths formed by the movements were regular curves with no abrupt changes. This assumption was verified by plotting the values of a representative sample of the curves in the range of interest. The second assumption was that the base of each of the prismatoids was parallel. With curved movement pathways this is not completely accurate, but by using small increments of $h$, the changes generated were observed to be of a value measurable only in the fourth decimal place.

Several safeguards were applied in the calculations of the volume to reduce errors. A very small increment of movement between calculations was established for the simulation which calculated the tracks as well as for the calculation of the incremental volumes. Approximately seventy-five steps in a $2-\mathrm{mm}$ lateral working side movement were used for each calculation of the total volume. In addition, the calculation of the volume of each prismatoid was independent of the others, reducing propagation of potential errors. An additional calculation was made for the solid containing the spherical outer surface of the total volume. Single and double precision calculations were compared as was varying the size of steps in the tracks and volumes. Double precision calculation did not significantly improve the accuracy of the study. Table 1 shows the identifying sign for volume.

\section{Action of the computer program}

The graphic program calculated paths of cusp movement. Instead of projecting them into three planes of visual display, groove elevation angles, coordinate plane angles and volumetric measurements were made. Data were collected and manipulated into two observation formats. In one, line graphs on $X-Y$ coordinates showed the relationship of a particular articulator setting or variation of tooth position to the resultant angle or volume change. The other format calculated the rate of change for the measured values. This latter format was expanded to show the rates of change in resultant tooth anatomy with the simultaneous manipulation of two experimental settings.

\section{Results}

The method employed described a simulated tooth. With a combination of computer programs, changes in morphology of the simulated tooth were observed as the articulator and control factors were changed. Various examples of resultant changes were demonstrated by graphs and tabulating the results.

In these examples, articulator end control changes were made bilaterally symmetrical and all other variables were maintained at the initial settings. Variations in the right 


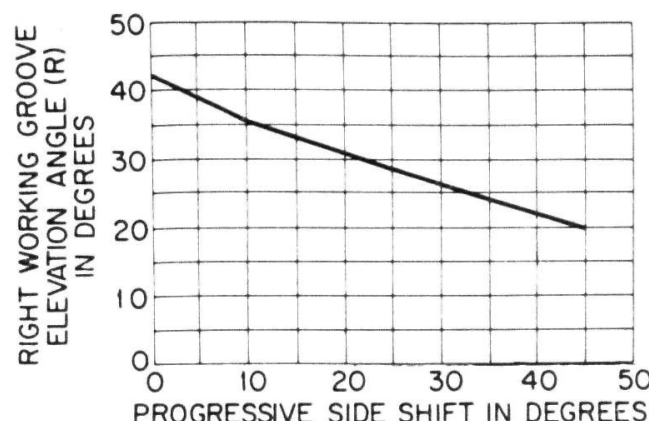

An increase in PSS results in a decrease in (R).

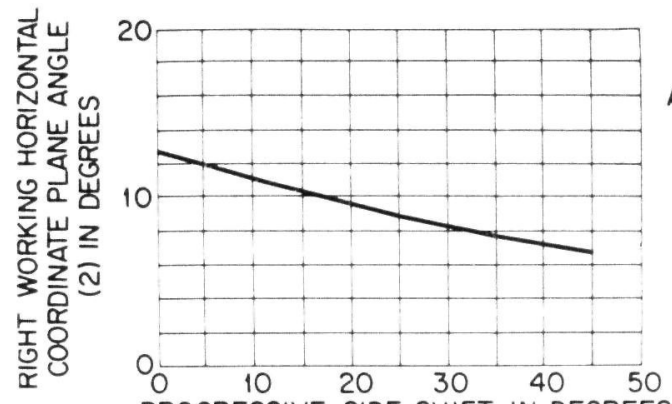

As PSS is increased (2) decreoses.
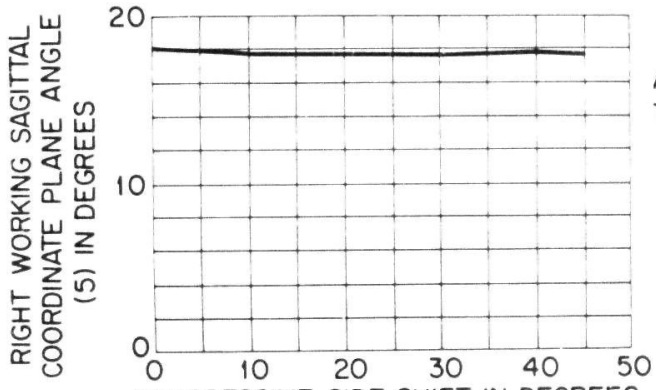

As PSS is increased almost no change occurs in (5) This groph shows a change of less than 0.50 degrees.

PROGRESSIVE SIDE SHIFT IN DEGREES
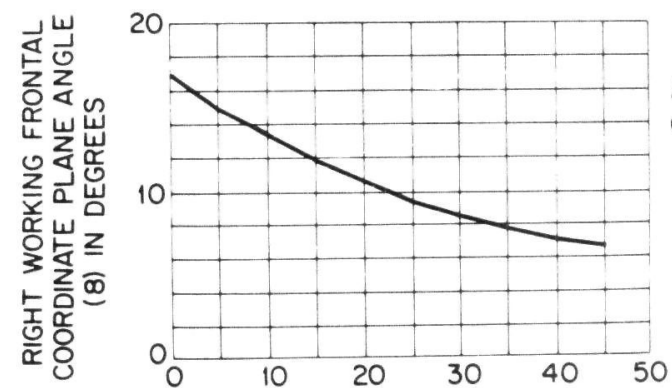

Agcin, as PSS decreoses its influence on the tooth onatomy is reflected as a decreose in angulation (8)

PROGRESSIVE SIDE SHIFT IN DEGREES
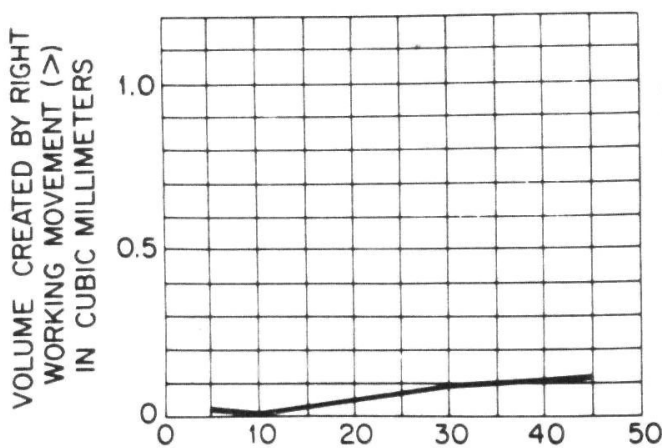

As the PSS approaches the initial setting of $10^{\circ}$, the volume decreases to zero Increasing PSS above $10^{\circ}$ shows a corresponding rise in volume. In spite of a lorge rise in PSS, the volume never exceeds .05 cubic millimeters.

Fig. 6. This series of five graphs aggregates results of tooth changes when progressive side shift was varied from $10^{\circ}$ to $45^{\circ}$. 


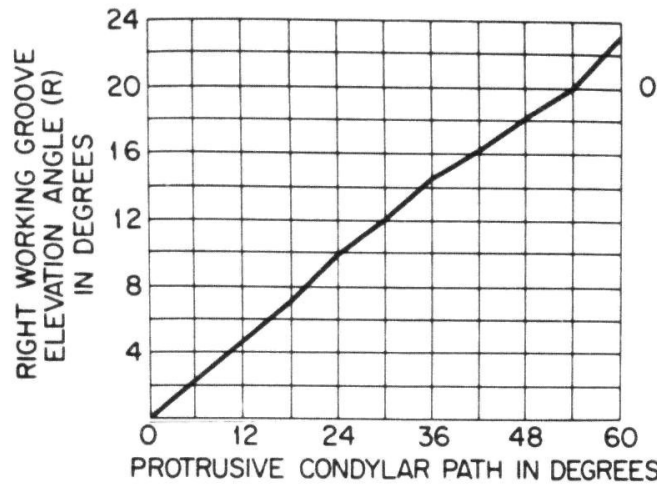

Once again os PCP is increased, $(R)$ increases.

秀

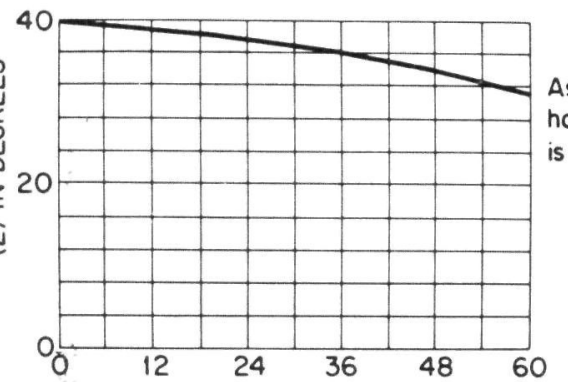

As PCP is increased from $0^{\circ}$ to $60^{\circ}$, the right working horizontal coordinate plane angle (2) decreased. This is the only instance in this study where PCP decreosed.

PROTRUSIVE CONDYLAR PATH IN DEGREES

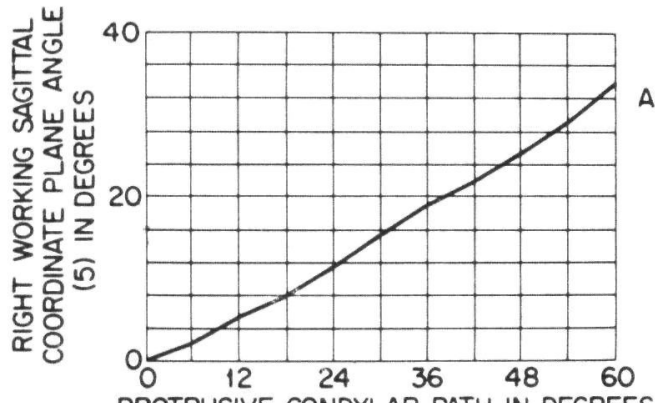

As PCP is increosed (5) decreoses

PROTRUSIVE CONDYLAR PATH IN DEGREES

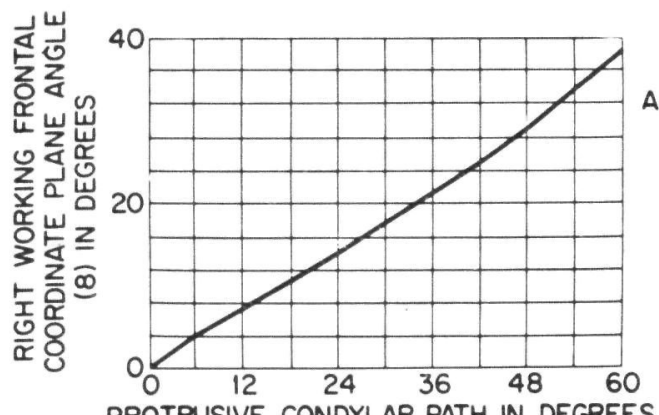

PROTRUSIVE CONDYLAR PATH IN DEGREES

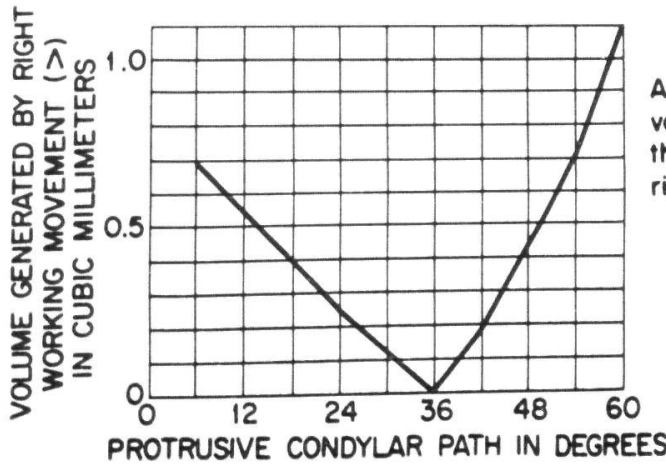

Fig. 7. This continued collection of results shows the effects of changes in protrusive condylar path from $0^{\circ}$ to $60^{\circ}$ on the geometry of the simulated tooth. 


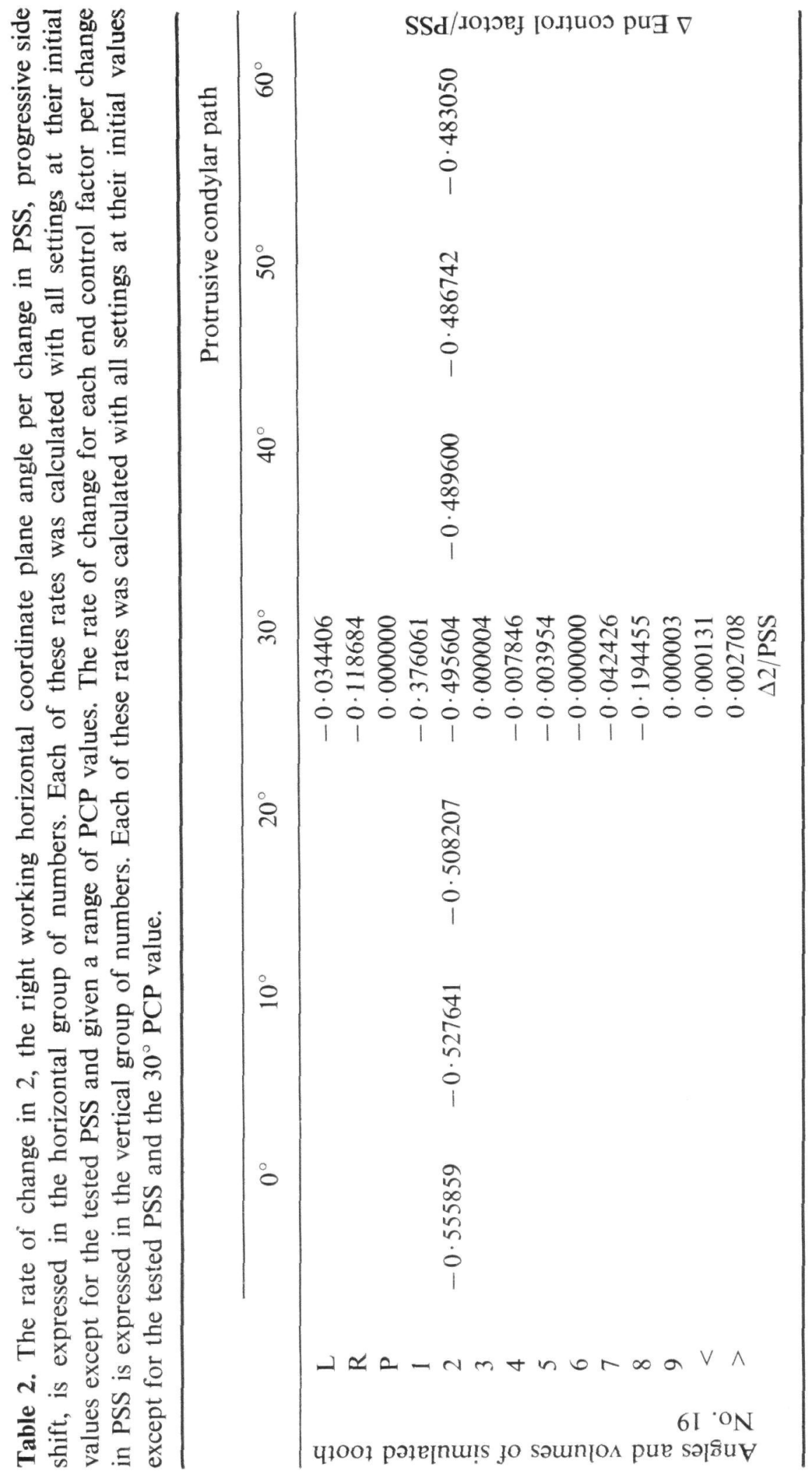


working path were examined. Results were collected for angular, linear and volumetric effects when the progressive side shift (PSS) was varied from $10^{\circ}$ to $45^{\circ}$. These changes in PSS are shown in Fig. 6 with graphs of horizontal, frontal, saggittal coordinate plane angle, the groove elevation angle and volume effects. Similarly, results were collected when the protrusive condylar path (PCP) was varied through the range from $0^{\circ}$ to $60^{\circ}$. For changes in PCP, Fig. 7 graphs horizontal, frontal and sagittal coordinate plane angle effects, as well as groove elevation angle and the right working volumetric effects.

The rate of change for each experimental circumstance was calculated. Table 2 lists the rate of change in each experimental setting where PSS was varied continuously through its range for each $10^{\circ}$ change in PCP, along with the correlation for the slope at the various PCP settings.

\section{Discussion}

The right working path of the mandibular left molar was expressed as angles from different coordinate planes and labelled R, 2, 5 and 8 .

When the effects of PCP were contrasted with those of PSS, it was noted that the horizontal plane is the only view where the angle of the right working path decreases when PCP is increased. Also, the horizontal plane is the only view where the rate of change of the PCP's effects on this working path are less than when the PSS is varied.

In contrasting the effects of PCP and PSS in the sagittal view, it was noted that the rate of change for PCP effects is nearly 500 times greater than the PSS effects. This is a vastly greater rate of change than any other calculated in this series and shows the dominance of PCP effects in the sagittal view.

When comparing the effects of PCP and PSS on volume, it can be seen that PCP effects the volume to a degree 150 times greater than that of PSS. It is interesting to note that as the end control values being varied approach the control values, the volume results approach zero.

A further examination of the rates of change for the sagittal/frontal expressions of the left molar's right working path is revealing. For example, when PCP is increased sequentially, the PSS rate of change for 5,8 and R, all increase sharply when PCP is increased from $0^{\circ}$ to $10^{\circ}$; they become more gradual thereafter. This seems to indicate that PCP is indispensable for articulator movement to correctly show the effects of PSS on frontal and sagittal views of the teeth. Additionally, the collection of incremental simultaneous changes could imply an effect of a curvature in the path of the condylar determinant. This needs further invstigation.

This brief sample of results shows a small portion of the findings which will be detailed in subsequent publications. In general, however, it can be said that end control factors can mutually affect one another, and the effects of end control factors can be seen in all views of the sample teeth. Further, the method of analysis which yields rates of change, in addition to basic numerical data, may prove to be a powerful comparative tool.

\section{Significance}

This computer simulation was developed to aid in understanding the relationship of positional condylar and incisal guidance factors to cuspid pathways and potential tooth morphology.

A standardization of the tooth as to its position, the direction from which it is 
viewed, the angles and distances recorded and a description of volumetric change can allow for inter-investigation comparisons which would otherwise be only grossly useful. With standardization of the model and accurate, sophisticated analytical observations of tooth changes, a variety of phenomenon can be investigated. Single or multiple parameters can be varied while still maintaining an orderly three-dimensional perspective. Their effects can be observed and conclusions made. The accuracy of this approach can allow both mechanically or clinically based observations to be carried out in a context of a useful standard of statistical significance. Through the use of this system as a teaching aid, the student of dental occlusion may be more easily taught to comprehend and predict directions of cuspal pathways, and the relative significances of compared changes. Additionally, by extrapolating from data secured from this simulation, useful equivalents or equations can be derived, allowing precise understanding without cumbersome analytical models.

\section{Acknowledgment}

This project was supported in part by Grant No. 4D08-PE01220 of the Department of Health, Education and Welfare, Bureau of Health Manpower Education.

\section{References}

Aull, A.E. (1965) Condylar determinants of occlusal patterns. Journal of Prosthetic Dentistry, 15, 826.

MJOR, P.S. (1965) The effect of the end controlling guidances of the articulator on cusp inclination. Journal of Prosthetic Dentistry, 15, 1055.

Roedema, W.H., Knapp, J.G, Spencer, J. \& Dever, M.K., (1978a) Computer-graphic representation of mandibular movements in three dimensions. I. The horizontal plane. Journal of Prosthetic Dentistry, 39, 378.

Roedema, W.H., Knapp, J.G., Spencer, J. \& Dever, M.K. (1978b) Computer-graphics representation of mandibular movements in three dimensions. II. The sagittal and frontal planes. Journal of Prosthetic Dentistry, 40, 385.

Manuscript accepted 19 October 1981 
This document is a scanned copy of a printed document. No warranty is given about the accuracy of the copy. Users should refer to the original published version of the material. 